\title{
Genetic variation for induced and basal resistance against leaf pathogen Pseudomonas syringae pv. tomato DC3000 among Arabidopsis thaliana accessions
}

\author{
Md Motaher Hossain ${ }^{1 *}$ (1) and Farjana Sultana²
}

\begin{abstract}
In Arabidopsis thaliana, significant efforts to determine the effect of naturally occurring variation between phenotypically divergent accessions on different biotic or abiotic stresses are underway. Although it is usually assumed that induced systemic resistance (ISR) against pathogen will covary with plant genetic variation, this assumption has not been tested rigorously in previous experiments. Here, we investigated heritable variation in resistance as well as Penicillium simplicissimum GP17-2-mediated ISR to the bacteria Pseudomonas syringae pv. tomato DC3000 (Pst) among a worldwide collection of accessions of $A$. thaliana. In this study, 75 Arabidopsis accessions were screened against the bacteria Pst following induction and non-induction treatment and their resistance levels were determined by measuring three components of $A$. thaliana resistance (infected leaf number, disease severity and pathogen growth). We observed extensive quantitative variation in the number of infected leaves, severity of disease symptoms and the bacterial population size among 75 accessions of $A$. thaliana infected with Pst. On the contrary, about a two-third of the accessions (49 accessions) showed a reduction in infected leaf number, disease severity and pathogen proliferation after treatment with GP17-2, indicating that GP17-2 induction of resistance is ecotype specific in Arabidopsis. The level of suppression was more pronounced for percent disease severity and pathogen proliferation than for number of infected leaves in ISR-inducible accessions. Accessions non-responsive to GP17-2 treatment generally appeared to be associated with higher basal resistance to infection by Pst. Future study with these parental lines employing a variety of crossing schemes may facilitate identification of major trait loci responsible for GP17-2-mediated ISR in Arabidopsis.
\end{abstract}

Keywords: Plant growth promoting fungus, Arabidopsis ecotypes, Natural variation, Basal resistance, Induced resistance

\section{Background}

Numerous studies have presented the phenomenon of induced resistance as an effective means to reduce damage of crops by various pathogens. Localized infection that results in protection in spatially separated parts of plants from an unrelated pathogen is called induced resistance or acquired resistance (Ross 1961a, b; Kuć 1987). Induced resistance could be local and systemic.

\footnotetext{
*Correspondence: hossainmm@bsmrau.edu.bd

${ }^{1}$ Department of Plant Pathology, Bangabandhu Sheikh Mujibur Rahman

Agricultural University, Gazipur 1706, Bangladesh

Full list of author information is available at the end of the article
}

Systemic induced resistance against pathogen lacks the specificity of the hormonal immune system, can be generated by a wide variety of structurally unrelated elicitors, and once activated; it is effective against a wide variety of organisms (Kuć 2006; Hossain et al. 2008). A multitude of potential responses occurs in plants following induction of systemic resistance. Complex sets of signals modulate a suite of responses within a plant to prevent an existing infection from spreading further or to combat secondary infections from a broad spectrum of pathogens. This multicomponent response requires a substantial commitment of cellular resources, including extensive genetic reprogramming and metabolic re-allocation (Somssich 
and Hahlbrock 1998). Hence, defenses are kept under tight genetic control and are activated only if the plant detects a prospective invader.

Non-pathogenic root colonizing fungi and their microbial metabolites have also shown to induce systemic resistance (ISR) as well against pathogens and insect herbivores. Plant growth promoting fungi (PGPF) are one such group that stimulates plant growth and yield of various crops (Hossain et al. 2008, 2014; Islam et al. 2014a, b). A wide variety of root-associated mutualistic fungi including species belonging to the genera Trichoderma, Fusarium, Penicillium, Phoma, Aspergillus and sterile fungi are reported to be PGPF (Koike et al. 2001; Hyakumachi and Kubota 2004; Hossain et al. 2008, 2014; Sultana et al. 2008; Horinouchi et al. 2011; Islam et al. 2014a, b). The PGPF isolate P. simplicissimum GP17-2 effectively controlled soil-borne diseases (Hyakumachi 1994) and also has been shown to induce systemic defense responses in cucumber plants against several diseases when applied as cell free filtrate (CF) (Shivanna et al. 1996; Koike et al. 2001). The 12,000 Da and lipid fraction in CF of $P$. simplicissimum GP17-2 were attributed for stimulating systemic defense responses in cucumber plants (Koike et al. 2001). Using the Arabidopsis thaliana-based model system, it has also been demonstrated that treatment with its $\mathrm{CF}$ is equally effective as living inocula in inducing ISR against the bacterial leaf pathogen Pseudomonas syringae pv. tomato DC3000 (Hossain et al. 2007). Arabidopsis is a model plant frequently used in molecular studies on plant-microbe interactions, including systemic acquired resistance and ISR. Some of the characteristics which have facilitated this research, are its small genome size $(125 \mathrm{Mb})$, a rapid life cycle (about 6 weeks), availability of a large number of mutant lines and wild ecotypes. The wild ecotypes, as a result of selection pressures imposed by their different environments of origin, show distinct variation in morphological and physiological traits. In fact, natural variation among genotypes is a prerequisite for biological effects of genetic diversity. The variation in morphological and physiological traits among plant genotypes may also result in variation in relative benefits and efficacy of induced resistance (Walters et al. 2011) and a greater understanding of these dynamic interactions is necessary to facilitate more effective use of elicitors for disease control.

To date, a variety of application methods has been considered to improve the integration of ISR into conventional agriculture, and in some cases with improved efficacy. Several researchers have suggested the need of breeding efforts focused on selection from natural variant ecotypes to add ISR to commercial cultivars. Data exist supporting heritability in the induction of plant defenses and a link between basal resistance and induced resistance (Ton et al. 2001a). This implies that plant genotypes differing in genetically determined basal resistance could differ in the extent to which induced resistance can be expressed. Therefore, it is important to know whether induction of systemic resistance by a particular strain contribute to the suppression of disease in genotypes ranging from susceptible to resistant. Until now only a few studies have examined genotypic effects of domesticated crops or their wild relatives on ISR (Walters et al. 2011). From $A$. thaliana model system, a great deal has already been known about the molecular mechanisms of induced resistance, mostly because of mutational screens. Yet, it is unclear what variability exists among the various ecotypes of $A$. thaliana for induced resistance responses, except in a limited case involving a single ISR-eliciting PGPR strain Pseudomonas fluorescens WCS417r, which is so far the only known such study (Van Wees et al. 1997; Ton et al. 1999). Moreover, ISR consequences of natural variation in plants have not yet been investigated using any PGPF. In the present study, we investigated heritable as well as inducible variation in resistance to the bacteria Ps. syringae among a wide-ranging collection of accessions of $A$. thaliana. In this study, we measured three components of host resistance (infected leaf number, disease severity and pathogen growth) and determined their relative importance to P. simplicissimum GP172-mediated ISR. Finally, analysis was done to find out the correlation between the ISR noninducible phenotype of Arabidopsis accessions and level of basal resistance against Pst. Screening of a set of 75 accessions in the current study for induced and basal resistance will be useful to appraise the variation in the interaction phenotypes of various Arabidopsis ecotypes observed upon pretreatment with GP17-2 and inoculation with Pst, opening the possibility of breeding plants with improved inducible resistance responses.

\section{Methods}

\section{Plant materials and growth condition}

A total of $75 \mathrm{~A}$. thaliana accessions was provided by $\mathrm{H}$. Koyama (Gifu University, Gifu, Japan) and used in the present study. The accessions were originally sourced from RIKEN Bioresource Center. The accession names were listed in Table 1, where the JA number is the stock number of the RIKEN Bioresource Center (Table 1). Arabidopsis plants were grown in hydroponic culture. Hydroponic culture was carried out using a culture apparatus, which was developed by Toda et al. (1999). This apparatus contains a plastic photo slide mount (Fuji film, Japan) and a mesh (50 holes per inch) made of thick nylon wire. The basic culture solution consisted of MGRL 
Table 1 Arabidopsis accessions screened for P. simplicissimum GP17-2-mediated ISR against Ps. syringae pv. tomato DC3000

\begin{tabular}{|c|c|c|c|c|c|}
\hline Serial number & Accession name & Stock number & Serial number & Accession name & Stock number \\
\hline 1 & $\mathrm{Aa}-0$ & JA1 & 39 & Dra-2 & JA292 \\
\hline 2 & $\mathrm{Ag}-0$ & $\mathrm{JA} 2$ & 40 & Edi-0 & JA69 \\
\hline 3 & $A k-1$ & JA3 & 41 & $\mathrm{El}-0$ & JA72 \\
\hline 4 & $A n-2$ & JA265 & 42 & $\mathrm{Ei}-2$ & JA70 \\
\hline 5 & $\mathrm{Ba}-1$ & JA8 & 43 & En-2 & JA73 \\
\hline 6 & Bay-0 & JA9 & 44 & Ep-0 & JA74 \\
\hline 7 & $\mathrm{Bd}-0$ & $J A$ & 45 & $\mathrm{Ge}-1$ & JA90 \\
\hline 8 & Be-0 & $\mathrm{JA} 12$ & 46 & Gr-6 & JA308 \\
\hline 9 & Be-1 & JA269 & 47 & $\mathrm{Hn}-0$ & JA108 \\
\hline 10 & Blh-0 & JA18 & 48 & Is-0 & JA111 \\
\hline 11 & Bla-1 & JA14 & 49 & Jl-5 & JA314 \\
\hline 12 & Bla-12 & JA16 & 50 & $\mathrm{Jm}-0$ & JA115 \\
\hline 13 & Bla-2 & JA270 & 51 & Kas-1 & JA119 \\
\hline 14 & Bla-3 & JA271 & 52 & $\mathrm{Kl}-5$ & JA124 \\
\hline 15 & $\mathrm{Br}-0$ & JA21 & 53 & La-1 & JA131 \\
\hline 16 & Bs-1 & JA22 & 54 & Ler-0 & NW20 \\
\hline 17 & Bs-5 & JA23 & 55 & Cvi-0 & N8580 \\
\hline 18 & Bsch-0 & JA24 & 56 & Ler-2 & N8581 \\
\hline 19 & Bsch-2 & $\mathrm{JA} 277$ & 57 & $\mathrm{Li}-2$ & JA135 \\
\hline 20 & Bu-0 & JA25 & 58 & Li-2-1 & JA136 \\
\hline 21 & Bu-14 & JA33 & 59 & Mh-0 & JA150 \\
\hline 22 & Bu-15 & JA35 & 60 & Ms-0 & JA157 \\
\hline 23 & Bu-17 & JA36 & 61 & Mt-0 & JA158 \\
\hline 24 & Bur-0 & JA44 & 62 & Old-2 & JA177 \\
\hline 25 & Cal-0 & JA48 & 63 & Oy-0 & JA181 \\
\hline 26 & Can-0 & JA49 & 64 & $\mathrm{~Pa}-1$ & JA182 \\
\hline 27 & Cha-0 & JA51 & 65 & $\mathrm{Pn}-0$ & JA190 \\
\hline 28 & Chi-0 & JA53 & 66 & Po-0 & JA349 \\
\hline 29 & Cit-0 & JA55 & 67 & Po-1 & JA191 \\
\hline 30 & $\mathrm{Cl}-0$ & JA56 & 68 & Ra-0 & JA195 \\
\hline 31 & Col-0 & JA58 & 69 & Rak-2 & JA196 \\
\hline 32 & Col-4 & N933 & 70 & $\mathrm{Rd}-0$ & JA197 \\
\hline 33 & Col-gl1 & N1644 & 71 & Rsch-4 & JA201 \\
\hline 34 & Da-0 & JA61 & 72 & UK-3 & $\mathrm{JA} 241$ \\
\hline 35 & Db-0 & JA62 & 73 & Van-0 & $\mathrm{JA} 243$ \\
\hline 36 & $\mathrm{Di}-0$ & JA63 & 74 & Ws-0 & JA252 \\
\hline 37 & Do-0 & JA66 & 75 & $X x x-0$ & JA261 \\
\hline 38 & Dr-0 & JA67 & & & \\
\hline
\end{tabular}

nutrients at a concentration of $10^{-1}$ (Fujiwara et al. 1992). Seeds of Arabidopsis were soaked in $0.5 \mathrm{~mL}$ of distilled water in Eppendorf tubes and kept in a refrigerator for 3 days at $4^{\circ} \mathrm{C}$ to synchronize germination. Thirty seeds, ten in each line were then placed on the apparatus. All apparatus were floated on $6 \mathrm{~L}$ of nutrient solution and kept in the growth room at $20^{\circ} \mathrm{C}$ with $14 \mathrm{~h}$ daytime and $10 \mathrm{~h}$ night cycles. The nutrient solution was renewed every 7 days and the culture was continued for 3 weeks.
The PGPF isolate $P$. simplicissimum GP17-2 and preparation of its CF

The PGPF P. simplicissimum GP17-2 was isolated from the rhizosphere of zoysiagrass (Zoysia tenuifolia L.). The fungus was grown in PDA media for 7 days. Twenty mycelial disks of GP17-2 culture were obtained from the growing margin of a colony on PDA and transferred to a $500 \mathrm{~mL}$ Erlenmeyer flask containing $200 \mathrm{~mL}$ potato dextrose broth (PDB). The fungus was 
cultured without shaking at room temperature $\left(25^{\circ} \mathrm{C}\right)$ for 10 days. The crude culture filtrate was separated from mycelia and filtered through two layers of Whatman No. 2 filter. The filtrate was then filter sterilized (0.22 $\mu \mathrm{m}$ Millipore filters, Millipore products division, Bedford, USA).

\section{Induction treatment of plants with CF from P. simplicissimum GP17-2}

Two-week old seedlings grown in hydroponic culture were treated with CF from P. simplicissimum GP172. Induction treatment of plants was performed 1 day before challenge inoculation by dipping the roots of seedlings in 50\% diluted CF of GP17-2 for $1 \mathrm{~h}$. Control plants were treated with $50 \%$ diluted PDB. Treated roots were washed thrice with sterilized distilled water and plants were returned to the hydroponic system with the same conditions as described above.

\section{Pathogen inoculation and disease assessment}

The rifampicin resistant virulent bacterium Ps. syringae pv. tomato DC3000 (Pst) provided by Y. Ichinose (Okayama University, Okayama, Japan) was used as pathogen for inoculating Arabidopsis plants. Plants were challenge inoculated 1 day after induction treatment with CF of GP17-2. The virulent bacterial pathogen Pst was cultured in liquid King's medium B (KB) at $28^{\circ} \mathrm{C}$. After overnight incubation, cells were collected by centrifugation, washed twice and re-suspended in $10 \mathrm{mM} \mathrm{MgSO}_{4}$. The plants were placed at $100 \%$ relative humidity 1 day before challenge inoculation. Plants were inoculated by spraying the Pst suspension containing, $2.5 \times 10^{7}$ cfu $\mathrm{mL}^{-1}$ bacteria and $0.01 \%(\mathrm{v} / \mathrm{v})$ Silwet L-77 (Nihon Unica, Tokyo, Japan) as a detergent on to the rosette leaves, until run off. The inoculated plants were kept at $100 \%$ relative humidity in darkness for 2 days in order for disease to develop. Plants were then transferred to a growth room. Five days after pathogen challenge, disease severity was measured for each plant by recording the percent infected leaves, percent of total plant leaf surface showing symptoms from $0=$ no symptoms to $100=$ most severe with necrotic symptoms (Ryu et al. 2003; Hossain et al. 2007). The number of Pst in inoculated leaves was assessed by collecting all leaves separately from three replications. Leaves were then weighed, rinsed thoroughly in sterile water and homogenized in sterilized distilled water (Pieterse et al. 1998; Hossain et al. 2007). Subsequently, appropriate dilutions were plated onto $\mathrm{KB}$ media supplemented with $50 \mathrm{mg} \mathrm{L}^{-1}$ rifampicin. After $48 \mathrm{~h}$ of incubation at $28^{\circ} \mathrm{C}$, the number of rifampicin-resistant colonyforming units (cfu) per gram of infected leaf tissue was determined.

\section{Statistical analysis}

The experimental design was completely randomized, consisting of three replications for each treatment. The experiment was repeated two to three times and treatment means obtained were separated using a Student's $t$ test. Susceptibility categorical data were expressed as number and percentages of accessions and were analyzed via Chi square $\left(\chi^{2}\right)$ analysis. Correlation coefficients as well as simple linear regression analysis were done by using the Analysis Tool Pak in Microsoft Excel. All analyses were done at $P<0.05$.

\section{Results}

Screening of Arabidopsis accessions for GP17-2-mediated ISR with regard to the percent infected leaves per plant

Five days after challenge with $P s t$ pathogen, the plants developed typical bacterial speck disease symptoms, consisting of necrotic or water-soaked spots surrounded by extensive chlorosis. Induced protection against the pathogen was quantified by determining the percent infected leaves per plant. Our results indicated a wide variation among the Arabidopsis accessions for percent infected leaves both as basal and induced resistance marker. As shown in Figure 1, percent infected leaves in control-treated plants varied over a range of $30-100 \%$ in the different accessions, where the lowest percentage of infected leaves was observed in Ra-0 and the highest was found in several accessions including Col-0. Similarly, accessions were differentially responsive to GP17-2 treatment in reducing the number of Pst-infected leaves compared to non-treated plants. Among the 75 accessions tested, 49 showed (65\%) a significant reduction in percent-infected leaves per plants. The percent-infected leaves in GP17-2-treated plant ranged from 24 to $99 \%$, where accession with the fewest percent infected leaves was En-0 and accession exhibiting greatest number of infected leaves was Cvi-0. In GP17-2-inducible ecotypes, the reduction in percent infected leaves was recorded to be $25-55 \%$ and was consistent in three independent experiments. The greatest reduction in percent infected leaves due to GP17-2 treatment was recorded in Bla-3 (55\%) and the lowest was in Bu-0 (Figure 2).

\section{Screening of Arabidopsis accessions for GP17-2-mediated ISR with regard to the percent disease severity per plant}

Results in Figure 3 show that percentage area of leaf with symptoms by the disease lesions (disease severity) varied across the non-treated and GP17-2-treated Arabidopsis accessions screened. The disease severity amongst controltreated accessions ranged from 12 to $100 \%$. There was a high severity of the disease on several accessions including Col-0, Be-1, Bla-1, Bla-3, Blh-1, Bu-17, Col-gl, Cvi-0, Pn-0, Uk-3, Van- 0 and Xxx-0 accession. The accessions showing the lowest disease severity was Dra- 2 . The severity of the 

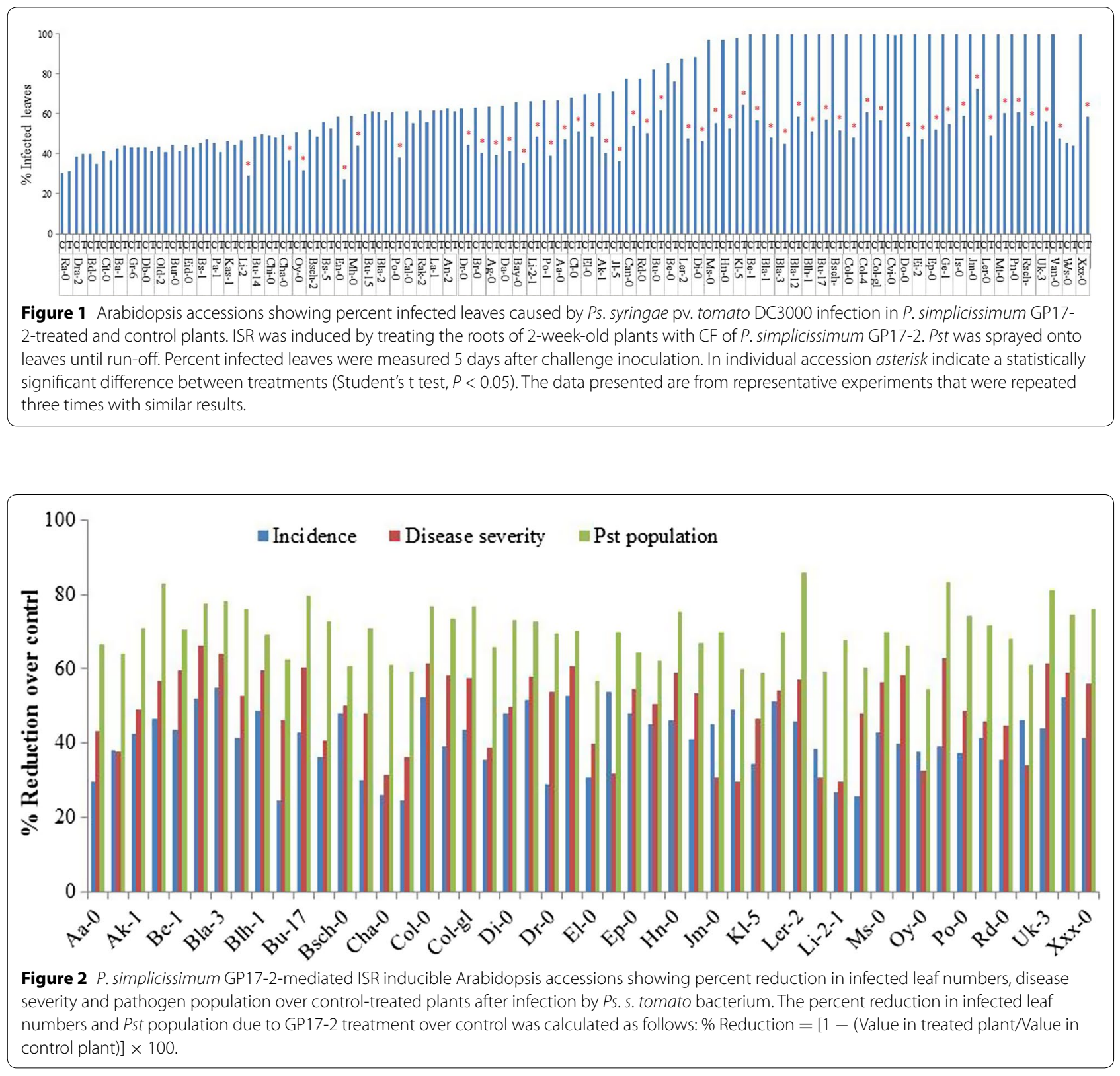

disease symptoms in many of these accessions were greatly reduced by GP17-2 treatment. Our results indicated that Arabidopsis accessions treated with GP17-2 were generally more resistant to foliar diseases caused by Pst and the disease severity in ISR-expressing and non-expressing plants ranged from 12 to $44 \%$. However, a total of 49 (65\%) GP172 -treated accessions exhibited a significant reduction in disease severity compared with non-induced control plants, showing a 29-66\% lower proportion of leaf surface with disease symptoms. Among these accessions, Bla-1 expressed the highest reduction in disease severity, while Jl-5 was the accession that exhibited the lowest reduction after GP17-2 treatment (Figure 2).

\section{Screening of Arabidopsis accessions for GP17-2-mediated ISR with regard to the foliar population of Pst}

Determination of the number of colony-forming units (cfu) of Pst in challenged leaves revealed that size of foliar population of Pst likewise varied among accessions both in control and GP17-2 treated plants. In control-treated accessions, Pst population ranged between $1.53 \times 10^{7}$ and $56.43 \times 10^{7} \mathrm{cfu} / \mathrm{g}$ leaves, where the highest Pst population was found in $\mathrm{Pn}-0$, while $\mathrm{Ba}-1$ exhibited the lowest (Figure 4). However, proliferation of Pst was significantly inhibited due to GP17-2 treatment in the same 49 accessions that showed significant reduction in infected leaf number and disease severity. Compared 


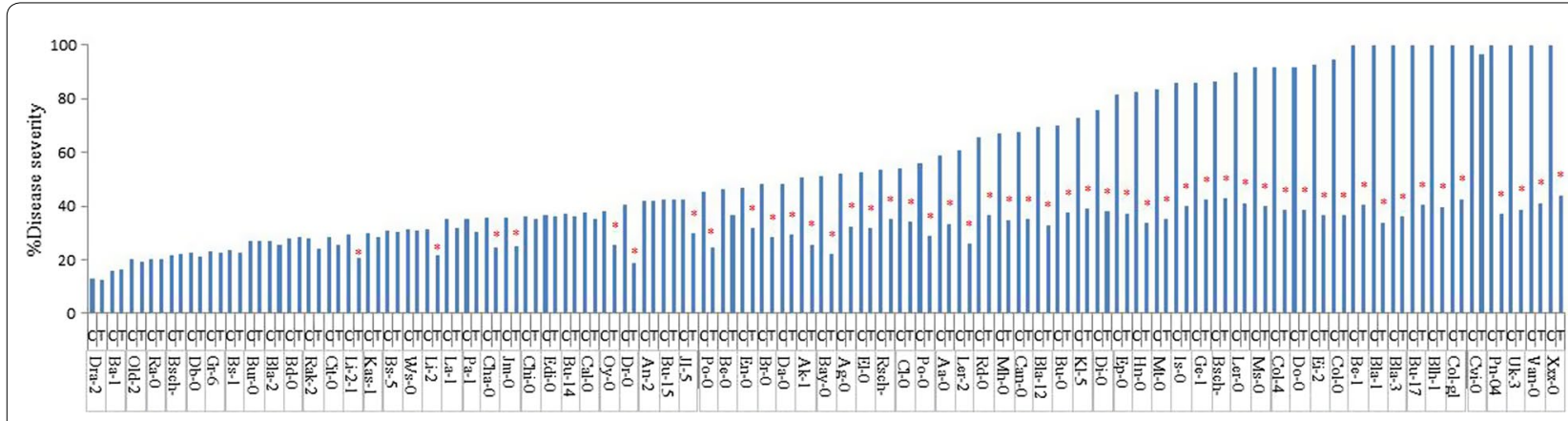

Figure 3 Arabidopsis accessions showing disease severity caused by Ps. syringae pv. tomato DC3000 infection in P. simplicissimum GP17-2-treated and control plants. ISR was induced by treating the roots of 2-week-old plants with CF of P. simplicissimum GP17-2. Pst was sprayed onto leaves until run-off. Disease severity was measured 5 days after challenge inoculation by recording the percentage of total leaf surface showing symptoms for each plant, where $0=$ no symptoms; $100=$ most severe necrotic symptoms. In individual accession asterisk indicate a statistically significant difference between treatments (Student's t test, $P<0.05$ ). The data presented are from representative experiments that were repeated three times with similar results.

with control-treated plants of these accessions, GP17-2treated plants showed a 53-84\% decrease in growth of Pst in challenged leaves, demonstrating that level of suppression was more pronounced for pathogen proliferation than for number of infected leaves and percent disease severity (Figure 2). Accession with maximum decrease in growth of Pst was Ler-2, while Oy-0 exhibited the lowest reduction. Clear differences in the inhibition of bacterial proliferation in leaves of GP17-2-treated plants indicate that symptom reduction is associated with inhibition of bacterial proliferation (Figure 4).

\section{Correlation between disease incidence or symptom severity and bacterial population}

Analysis was made to determine how far expression of GP17-2-mediated ISR depends on the level of basal resistance in Arabidopsis ecotypes to Pst. Percent infected leaves and symptom severity due to Pst infection are equivalent phenotypic indicators of host basal resistance to the bacterium. When a large number of accessions is used, the estimation of infected leaf number will considerably save time compared to that of disease severity. However, the variation in basal resistance with respect to disease severity was much wide ranging than to number of infected leaves. Therefore, disease severity provides more appropriate phenotype of basal resistance in ecotypes to Pst infection. Based on the mean disease severity in control-treated plants, individual accession was hypothetically categorized as either highly susceptible, moderately susceptible (70-40\%) or lowly susceptible $(<40 \%)$. Infection phenotypes with disease severity $>70 \%$ were classified as highly susceptible and those with disease severity of $70-40 \%$ and $<40 \%$ were classified as moderately and lowly susceptible, respectively (Table 2). Out of 75 accessions evaluated, 25 accessions were found to fall within highly susceptible group. Of the remaining accessions, 23 accessions were moderately susceptible and 27 were lowly susceptible. Among the 25 highly susceptible accessions, 24 (96\%) showed significant level of GP17-2-mediated ISR against Pst, when disease severity

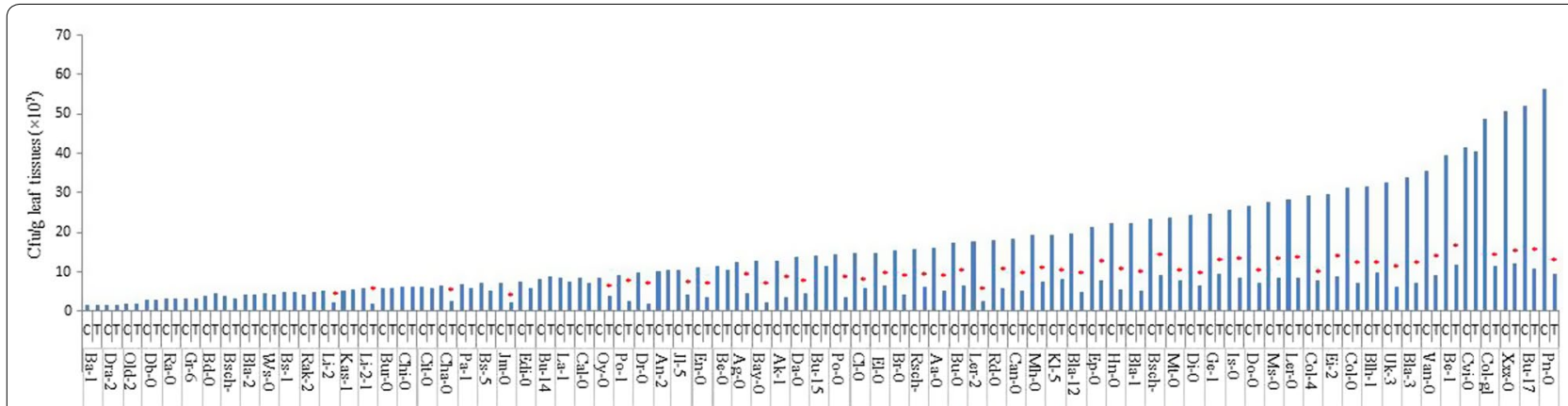

Figure 4 Arabidopsis accessions showing number of Ps. syringae tomato bacterium in leaves of $P$. simplicissimum GP17-2-treated and control plants. Data are presented as numbers of $\mathrm{cfu}^{-1}$ fresh weight, each from three sets of eight whole shoots harvested 5 days after challenge inoculation with virulent pathogen Pst. In individual accession asterisk indicate a statistically significant difference between treatments (Student's t test, $P<0.05)$. The data presented are from representative experiments that were repeated twice with similar results. 
Table 2 Response of Arabidopsis accessions to Ps. syringae pv. tomato DC3000 infection and P. simplicissimum GP172-mediated ISR

\begin{tabular}{|c|c|c|c|c|c|c|}
\hline $\begin{array}{l}\text { Groups (based on } \\
\text { percent disease severity) }\end{array}$ & $\begin{array}{l}\text { Number of } \\
\text { accessions }\end{array}$ & $\begin{array}{l}\text { Number of ISR } \\
\text { responsive accessions }\end{array}$ & $\begin{array}{l}\text { \% Accessions } \\
\text { expressing ISR }\end{array}$ & $\begin{array}{l}\text { Chi square } \\
\text { test }\left(\chi^{2}\right)\end{array}$ & df & $P$ value ${ }^{a}$ \\
\hline $\begin{array}{l}\text { Highly susceptible } \\
\text { (DS }>70 \%)\end{array}$ & 25 & 24 & 96.00 & 10.36 & 2 & 0.0056 \\
\hline $\begin{array}{l}\text { Moderately susceptible } \\
\quad(\mathrm{DS}=40-70 \%)\end{array}$ & 23 & 20 & 86.95 & & & \\
\hline $\begin{array}{l}\text { Lowly susceptible } \\
\text { (DS <40\%) }\end{array}$ & 27 & 5 & 18.51 & & & \\
\hline
\end{tabular}

was compared between GP17-2-treated and non-treated plants (Table 2). Similarly, out of 23 moderately susceptible accessions group, 20 (86.95\%) showed significantly reduced disease severity in GP17-2-treated plants compared to non-treated plant (Table 2). On the other hand, only $5(18.5 \%)$ of 27 lowly susceptible accessions were responsive to GP17-2-treatment in showing significant level of ISR against the bacterium (Table 2). These indicate that most plants have higher expression of GP172 -mediated ISR despite higher degrees of infection. The Chi Square $\left(\chi^{2}=10.36 ; \mathrm{df}=2 ; 0.001<P<0.010\right)$ test revealed a significant difference in ISR-responsiveness among different Pst susceptibility accession groups in Arabidopsis. In other words, ISR-responsiveness was not equally distributed across the different levels of host susceptibility in Arabidopsis accessions towards Pst. Accessions that had higher Pst susceptibility were likely to be more ISR-responsive than accessions that had less susceptibility. In order to elucidate a clear functional relationship between the host basal resistance and the GP17-2-induced ISR responsiveness of Arabidopsis ecotypes, we calculated the correlation between the symptom severity in control-treated ecotypes and the reduction in symptom severity in GP17-2-treated plants. There was a significant positive relationship observed between the symptom severity in control-treated ecotypes and the reduction in symptom severity due to GP17-2 treatment in the same plants, $\mathrm{r}(223)=0.84$, $P<0.000$. That means percent reduction in symptom severity in ISR-inducible ecotypes linearly increased as percent disease severity increased in control-treated plants. Among all accessions, $70 \%$ of the values fit the model (Figure 5). These results indicate the ISR-inducible phenotype of Arabidopsis ecotypes was highly correlated with a relatively low level of basal resistance against $P s t$.

\section{Discussion}

The capacity to express $P$. simplicissimum GP17-2 mediated ISR was found to be dependent on the plant genotype. Selection of naturally occurring variants that were

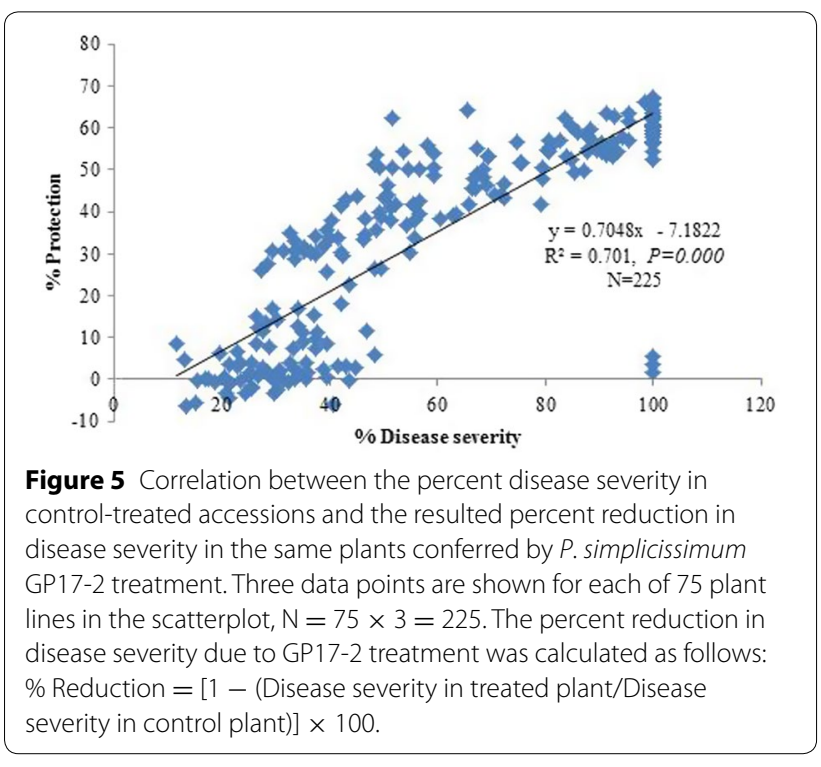

higher resistant to disease could be useful for future breeding program. Useful genes for plant breeding are already abundant. In the present study, 75 different Arabidopsis ecotypes were screened to develop an understanding of naturally occurring variation in ISR inducibility and basal resistance against Pst. From our results, it clearly appears that the number of Pst-infected leaves, the proportion of leaf surface area with the disease symptom and the size of Pst population in infected leaves were equivalent phenotypic indicators of both inducible and basal host resistance to the bacterium. Arabidopsis ecotypes were differentially responsive in expressing basal resistance as well as the GP17-2-mediated ISR, when compared these phenotypic indicators during infection by Pst bacterium. The ecotypes ranged from highly susceptible to highly resistant to Pst infection. Likewise, they were responsive to completely non-responsive to GP17-2 treatment in reducing Pst infection, suggesting that specific interactions between the GP17-2 and the plant ecotypes determine ISR in Arabidopsis. For instance, 49 of the 
tested ecotypes including Col- 0 and Ler- 0 were responsive to induction of ISR by GP17-2, resulting in a significant suppression of the disease caused by Pst in these plants. In ISR-inducible ecotypes, although induced resistance was expressed as a reduction in infected leaf numbers, symptom severity and Pst proliferation, the level of reduction varied for each ISR trait. Our results indicate that GP172-mediated ISR had more severe effect on Pst population than in infected leaves and disease severity. On the contrary, 27 ecotypes including Cvi-0 did not develop ISR after treatment with GP17-2. This observation commemorates a previous conclusion that capacity to trigger ISR is ecotype-dependent in Arabidopsis (Ton et al. 1999, 2001a, b; Van Wees et al. 1997). In that study, ISR elicited by Ps. fluorescence WCS417r against Pst was clearly associated with ecotype-specific. In carnation, cultivar specificity with regard to expression of WCS417r-mediated ISR has also been reported (Van Peer et al. 1991). Rhizobacteria-mediated ISR against Pst in Arabidopsis required the presence of a single dominant gene and the corresponding locus, designated as ISR 1 was mapped between markers B4 and GL1 on chromosome 3 (Ton et al. 1999). Therefore, the observed non-responsiveness in 27 ecotypes to express GP17-2-mediated ISR suggests the absence of (a) genetic determinant(s) essential for induction and expression of ISR.

The level of basal resistance can influence the extent to which GP17-2-mediated ISR is expressed. To investigate how far expression of GP17-2-mediated ISR depends on the level of basal resistance understand the relationship between ISR responsiveness and basal resistance to pathogen, we classified the accessions arbitrarily on the basis of disease severity. In these groupings, majority (95.4\%) of highly susceptible accessions were ISR responsive, ruling out the possibility that ISR was masked by the high susceptibility of these ecotypes to Pst. On the other hand, highly resistant ecotypes were commonly ISRnoninducible, meaning that expression of GP17-2-mediated ISR is less consistent when basal resistance of these ecotypes to Pst is high. These results are in agreement with previously obtained results of Liu et al. (1995) who demonstrated that two susceptible cucumber cultivars expressed ISR after treatment with Serratia marcescens 90-166, while a resistant cultivar did not. Likewise, the WCS417r non-induced Ws plants infected with Phytophthora parasitica did not exhibit enhanced disease susceptibility compared to ISR-expressing Col-0 (Ton et al. 2001b). Therefore, the observed phenomenon that Arabidopsis ecotypes capable of expressing ISR have a substantially lower level of basal resistance to Pst than ecotypes impaired in their ISR response strongly suggests that GP17-2-mediated ISR in Arabidopsis does not utilizes components of the basal resistance pathway. Apparently, both defense mechanisms are genetically distinct resistance responses. However, several situations have been reported in which an opposing association between the capacity to express ISR and the level of basal resistance against the challenging pathogen was existed. For instance, ISR induced by WCS417 $r$ against fusarium wilt, caused by Fusarium oxysporum f. sp. dianthi, was clearly expressed in the moderately resistant cultivar Pallas, but less strongly and consistently in the susceptible cultivar Lena (Van Peer et al. 1991). Similarly, the WCS417rmediated ISR-noninducible phenotype in Arabidopsis correlated with a relatively low level of basal resistance against Pst (Ton et al. 2001b). Moreover, both susceptible and resistant cultivars of radish were capable of expressing Ps. fluorescens WCS374-mediated ISR against fusarium wilt to the same extent (Leeman et al. 1995). These demonstrate that a clear correlation between the capacity to express ISR and the level of basal resistance against the challenging pathogen was absent in many instances. However, most of the aforementioned studies used only a limited number of cultivars or ecotypes in contrast to the present study which had done a more comprehensive analysis using a large number of ecotypes and delivered a definitive understanding of their naturally occurring variation in ISR inducibility and basal resistance against Pst.

In this study, we studied ecotype specificity of GP172-mediated ISR in Arabidopsis in relation to the level of ecotype-specific basal resistance against Pst. The GP172-nonresponsive phenotype correlates with a relatively high level of basal resistance against Pst. These studies prepared the ground for future studies to clearly show the mechanistic response of resistance or susceptibility in these accessions against $P$. syringae. Further studies in the genetic analysis of variation in the ISR expression ability may contribute to a better understanding of its role in affecting quantitative trait variation at the phenotypic level.

\section{Conclusion}

Plant growth promoting fungus $P$. simplicissimum GP17-2 induction of resistance against the leaf pathogen Ps. s. pv. tomato DC3000 (Pst) was ecotype specific in Arabidopsis. The GP17-2- nonresponsive phenotype correlated with a relatively high level of basal resistance against Pst. Accessions non-responsive to GP17-2 treatment were generally appeared to be associated with higher basal resistance to infection by Pst. This study will help exploit genetic variability within populations of Arabidopsis to improve the utility of ISR in the field.

\section{Abbreviations}

PGPF: plant growth promoting fungi; ISR: induced systemic resistance; Pst: Pseudomonas syringae pv. tomato DC3000. 


\section{Authors' contributions}

$\mathrm{MH}$ has contributed to the experimental conception and design, to the implementation of all experiments, to the acquisition of data, to the analysis and interpretation of data. $\mathrm{MH}$ is also involved in drafting and revising the manuscript. FS has mainly deterred the bacterial population in leaves, contributed to the acquisition of data and also revised the manuscript. Both authors read and approved the final manuscript.

\section{Author details}

1 Department of Plant Pathology, Bangabandhu Sheikh Mujibur Rahman Agricultural University, Gazipur 1706, Bangladesh. ${ }^{2}$ College of Agricultural Sciences, International University of Business Agriculture and Technology, Dhaka 1230, Bangladesh.

\section{Acknowledgements}

We would like to thank H. Koyama for providing Arabidopsis seeds. We thank provision of bacterial pathogen by Y. Ichinose. Financial support through the Ministry of Education, Culture, Sports, Science and Technology (MONBUKAGAKUSHO), Japan is greatly acknowledged.

\section{Compliance with ethical guidelines}

\section{Competing interests}

The authors declare that they have no competing interests.

Received: 14 March 2015 Accepted: 28 May 2015

Published online: 26 June 2015

\section{References}

Fujiwara T, Hirai YM, Chino M, Komeda Y, Naito S (1992) Effect of sulfur nutrition on expression of soybean seed storage protein genes in transgenic petunia. Plant Physiol 99:263-268

Horinouchi H, Watanabe H, Taguchi Y, Muslim A, Hyakumachi M (2011) Biological control of Fusarium wilt of tomato with Fusarium equiseti GF191 in both rock wool and soil systems. Biol Control. 56:915-923

Hossain MM, Sultana F, Kubota M, Koyama H, Hyakumachi M (2007) The plant growth-promoting fungus Penicillium simplicissimum GP17-2 induces resistance in Arabidopsis thaliana by activation of multiple defense signals. Plant Cell Physiol 48:1724-1736

Hossain MM, Sultana F, Kubota M, Hyakumachi M (2008) Differential inducible defense mechanisms against bacterial speck pathogen in Arabidopsis thaliana by plant-growth-promoting-fungus Penicillium sp. GP16-2 and its cell free filtrate. Plant Soil 304:227-239

Hossain MM, Sultana F, Miyazawa M, Hyakumachi M (2014) The plant growthpromoting fungus Penicillium spp. GP15-1 enhances growth and confers protection against damping-off and anthracnose in the cucumber. J Oleo Sci 63:391-400

Hyakumachi M (1994) Plant-growth-promoting fungi from turf grass rhizosphere with potential for disease suppression. Soil Microorg 44:53-68

Hyakumachi M, Kubota M (2004) Fungi as plant growth promoter and disease suppressor. In: Arora DK (ed) Fungal biotechnology in agricultural, food, and environmental applications. Marcel Dekker, New York, USA

Islam S, Akanda AM, Prova A, Sultana F, Hossain MM (2014a) Growth promotion effect of Fusarium spp. PPF1 from bermudagrass (Cynodon dactylon) rhizosphere on Indian spinach (Basella alba) seedlings are linked to root colonization. Arch Phytopathol Plant Protect 47:2319-2331

Islam S, Akanda AM, Sultana F, Hossain MM (2014b) Chilli rhizosphere fungus Aspergillus spp. PPA1 promotes vegetative growth of cucumber (Cucumis sativus) plants upon root colonization. Arch Phytopathol Plant Protect 47:1231-1238

Koike N, Hyakumachi M, Kageyama K, Tsuyumu S, Doke N (2001) Induction of systemic resistance in cucumber against several diseases by plant growth-promoting fungi: lignification and superoxide generation. Eur J Plant Pathol 107:523-533

Kuć J (1987) Plant immunization and its applicability for disease control. In: Chat I (ed) Innovative approaches to plant disease control. Wiley, New York, USA
Kuć J (2006) What's old and what's new in concepts of induced systemic resistance in plants, and its application. In: Tuzun S, Bent E (eds) Multigenic and induced systemic resistance in plants. Springer Science + Business Media, Inc., New York, USA

Leeman M, Van Pelt JA, Den Ouden FM, Heinsbroek Bakker M, Schippers B (1995) Biocontrol of fusarium wilt of radish by lipopolysaccharides of Pseudomonas fluorescens. Phytopathology 85:1021-1027

Liu L, Kloepper JW, Tuzun S (1995) Induction of systemic resistance in cucumber by plant growth-promoting rhizobacteria: duration of protection and effect of protection and root colonization. Phytopathology 85:1064-1068

Pieterse CMJ, Wees SCM, Pelt JA, Knoester M, Laan R, Gerrits H et al (1998) A novel signaling pathway controlling induced systemic resistance in Arabidopsis. Plant Cell 10:1571-1580

Ross AF (1961a) Localized acquired resistance to plant virus infection in hypersensitive hosts. Virology 14:329-339

Ross AF (1961 b) Systemic acquired resistance induced by localized virus infections in plants. Virology 14:340-358

Ryu C-M, Hu C-H, Reddy MS, Kloepper JW (2003) Different signaling pathways of induced resistance by rhizobacteria in Arabidopsis thaliana against two pathovars of Pseudomonas syringae. New Phytol 160:413-420

Shivanna MB, Meera MS, Hyakumachi M (1996) Role of root colonization ability of plant growth promoting fungi in the suppression of take-all and common root rot of wheat. Crop Protect 15:497-504

Somssich I, Hahlbrock K (1998) Pathogen defense in plants—a paradigm of biological complexity. Trends Plant Sci 3:86-90

Sultana F, Hossain MM, Kubota M, Hyakumachi M (2008) Elicitation of systemic resistance against the bacterial speck pathogen in Arabidopsis thaliana by culture filtrates of plant growth-promoting fungi. Can J Plant Pathol 30:196-205

Toda T, Koyama H, Hara T (1999) A simple hydroponic culture method for the development of a highly viable root system in Arabidopsis thaliana. Biosci Biotechnol Biochem 63:210-221

Ton J, Pieterse CMJ, van Loon LC (1999) Identification of a locus in Arabidopsis controlling both the expression of rhizobacteria-mediated induced systemic resistnace (ISR) and basal resistance against Pseudomonas syringae pv. tomato. Mol Plant-Microb Interact 12:911-918

Ton J, Davison S, Van Loon LC, Pieterse CMJ (2001a) Heritability of rhizobacteria-mediated induced systemic resistance and basal resistance in Arabidopsis. Eur J Plant Pathol 107:63-68

Ton J, Davison S, van Wees SCM, van Loon LC, Pieterse CMJ (2001 b) The Arabidopsis ISR1 locus controlling rhizobacteria-mediated induced systemic resistance is involved in ethylene signaling. Plant Physiol 125:652-661

Van Peer R, Niemann GJ, Schippers B (1991) Induced resistance and phytoalexin accumulation in biological control of Fusarium wilt of carnation by Pseudomonas sp. strain WCS417r. Phytopathology 81:728-734

van Wees SCM, Pieterse CMJ, Trijssenaar A, van't Westende YAM, Hartog F, van Loon LC (1997) Differential induction of systemic resistance in Arabidopsis by biocontrol bacteria. Mol Plant-Microb Interact 6:716-724

Walters DR, Havis ND, Paterson L, Taylor J, Walsh DJ (2011) Cultivar effects on the expression of induced resistance in spring barley. Plant Dis 95:595-600

\section{Submit your manuscript to a SpringerOpen ${ }^{\odot}$ journal and benefit from:}

- Convenient online submission

- Rigorous peer review

- Immediate publication on acceptance

- Open access: articles freely available online

- High visibility within the field

- Retaining the copyright to your article

Submit your next manuscript at $\boldsymbol{s p r i n g e r o p e n . c o m ~}$ 